Service social

\title{
Les praticiens sociaux et leur code de déontologie
}

\section{René Auclair}

Volume 40, numéro 1, 1991

\section{Éthique et intervention sociale}

URI : https://id.erudit.org/iderudit/706515ar

DOI : https://doi.org/10.7202/706515ar

Aller au sommaire du numéro

\section{Éditeur(s)}

École de service social de l'Université Laval

\section{ISSN}

1708-1734 (numérique)

Découvrir la revue

Citer cet article

Auclair, R. (1991). Les praticiens sociaux et leur code de déontologie. Service social, 40(1), 71-90. https://doi.org/10.7202/706515ar

\section{Résumé de l'article}

Dans cet article, l'auteur présente les résultats d'une recherche effectuée dans le cadre du cours de Déontologie professionnelle du programme de baccalauréat en service social de l'Université Laval, au trimestre d'hiver 1990, avec la participation des 155 étudiantes et étudiants sortants.

Une introduction théorique tente, dans un premier temps, de situer le problème et de dégager une stratégie d'analyse. Cette stratégie consiste essentiellement à considérer l'activité des corporations professionnelles dans une perspective historique.

Les praticiens sociaux eux-mêmes sont appelés, dans un second temps, à s'exprimer en entrevue sur les questions de déontologie professionnelle.

Nous retiendrons de ces entrevues qu'en général les praticiens sociaux connaissent leur code de déontologie de façon superficielle, et que cela tiendrait au fait que leur formation éthique formelle est limitée. Ils font plutôt rarement usage de leur code, ne le font pas connaître explicitement à leurs clients. Enfin, même s'ils doutent de son efficacité, ils continuent de croire à la nécessité du code de déontologie. 
René Auclair, professeur, École de service social, Université Laval.

\section{Les praticiens sociaux et leur code de déontologie}

René Auclair

\section{Introduction}

Le présent article a pour objectif de faire connaître les résultats d'une recherche effectuée dans le cadre du cours de Déontologie professionnelle du programme de baccalauréat en service social de I'Université Laval au trimestre d'hiver 1990, avec la participation des 155 étudiants sortants ${ }^{1}$.

En dépit du fait que des recherches aient été effectuées sur la déontologie dans le domaine du service social, la présente étude pourrait être la première au Québec à examiner ce que les praticiens sociaux ${ }^{2}$ connaissent vraiment de l'éthique professionnelle.

La question de l'éthique professionnelle est vaste et complexe et nous pouvons l'aborder sous plusieurs angles. À première vue, I'analyse de questions de déontologie professionnelle peut sembler relever avant tout de la morale et du droit : la morale professionnelle et les codes de déontologie qui la formalisent se présentent tout naturellement comme objets de réflexion pour ces deux disciplines. II y a cependant d'autres types d'analyse - historique, sociologique, économique - , qui peuvent venir jeter un éclairage intéressant sur le sujet.

En ce qui nous concerne, nous ne pouvions évidemment pas faire le tour du sujet. C'est pourquoi nous nous sommes inspirés, pour réaliser notre étude, de la méthodologie développée par l'équipe de recherche de l'Institut supérieur des sciences humaines dans leur rapport de recherche préparé à la demande de l'Office des professions du Québec en janvier $1977^{3}$.

Une introduction théorique tente, dans un premier temps, de situer le problème et de dégager une stratégie d'analyse. Cette stratégie consiste essentiellement à considérer l'activité des corporations professionnelles dans une perspective historique. 
Les praticiens sociaux eux-mêmes sont appelés, dans un second temps, à s'exprimer en entrevue sur les questions de déontologie professionnelle.

Finalement, il faut noter que ce travail comporte certaines limites : d'abord le fait de ne pas proposer d'analyses juridique ni économique restreint largement notre vision de l'éthique professionnelle dans le contexte actuel. À cela il faut ajouter le caractère exploratoire de notre étude qui ne nous permettait pas des analyses qu'il nous aurait semblé pertinent de faire mais auxquelles nous avons été contraints de renoncer, faute de temps.

\section{Cadre de référence et problématique}

\section{L'activité des corporations professionnelles vue dans une pers- pective historique $\mathrm{e}^{4}$}

Le fait que l'ancienneté des corporations soit la caractéristique la plus intensément reliée à leur niveau d'activité en matière de protection du public fournit une piste fort intéressante dans l'interprétation des résultats de l'analyse. II est possible, en effet, d'avancer I'hypothèse que les corporations sont constituées à des époques données pour répondre à des besoins propres à ces époques. C'est ce que semble confirmer la polarisation des corporations autour de deux moments historiques de l'évolution des activités de travail :

- les corporations constituées depuis plus de cinquante ans sont habituellement composées de membres exerçant majoritairement en pratique privée, détiennent un exercice exclusif, disposent de revenus plus importants et exercent à un plus haut niveau les activités de protection du public qui constituent les quatre indicateurs; - les corporations constituées plus récemment sont habituellement composées de personnes n'exerçant pas à leur propre compte, ne détiennent pas d'exercice exclusif, disposent de revenus moins élevés et exercent peu les activités de protection du public concernées.

Les différences observées entre les deux groupes de corporations semblent attester que le mécanisme de la corporation professionnelle n'a pas su, au cours des ans, s'adapter vraiment à l'évolution des conditions sociales et plus particulièrement à l'évolution du monde du travail. Aussi, il importe d'en retracer les origines, d'examiner les facteurs qui causent ses difficultés d'adaptation et de chercher pourquoi il exerce toujours autant d'attrait auprès des producteurs de services. 


\section{Le corporatisme professionnel traditionnel}

Les corporations professionnelles constituées au Québec au cours de la seconde moitié du $\mathrm{XIX}^{\mathrm{e}}$ siècle et au début du $\mathrm{XX}^{\mathrm{e}}$ siècle s'inscrivaient dans un contexte économique fondé sur la libre entreprise. Les producteurs de biens et de services étaient le plus souvent éparpillés dans des entreprises de petite dimension et la plupart des professionnels exerçaient en cabinet privé, seuls, autonomes et isolés.

Le plus souvent, les membres des corporations constituées à cette époque ont occupé des champs de connaissances étendus et généraux. La main-d'œuvre qualifiée était rare, la somme des connaissances était moindre qu'aujourd'hui, la division du travail n'était pas aussi poussée. À chaque champ de connaissances correspondait un champ d'exercice - médecine, médecine dentaire, droit, etc. - et chaque professionnel pouvait offrir un service couvrant la totalité de ce champ.

Dans ce contexte, la création de corporations professionnelles répondait à des besoins précis, aussi bien du professionnel que du client : besoin d'identification du professionnel isolé à un groupe homogène; besoin de standardisation des services offerts par des professionnels autonomes à des clients peu aptes à en apprécier la qualité, etc. La constitution d'une corporation permettait en quelque sorte de stabiliser la distribution des services professionnels dans un secteur donné tout en respectant le régime de libre entreprise qui prédominait à l'époque.

Soucieux à la fois de la défense des intérêts de leurs membres, de la promotion de la profession (développement des connaissances et des techniques) ainsi que du bien-être de leur clientèle et du public en général (idéal de service), ces corporations autonomes constituaient le seul mécanisme de contrôle des professions en cause. II paraissait difficile de régir autrement des producteurs autonomes exerçant individuellement et détenant des monopoles sur de vastes champs de connaissances. Ainsi fut délégué aux seuls producteurs de services le soin de veiller aux intérêts des consommateurs de ces mêmes services.

Cette situation a conduit les corporations à l'élaboration d'une idéologie professionnelle fondée sur l'autodiscipline et le sens éthique de leurs membres. En affirmant la préséance de l'intérêt du public sur l'intérêt du professionnel, cette idéologie visait à réduire le caractère ambivalent du corporatisme professionnel basé sur l'autoréglementation. Elle reposait sur la notion de vocation ou de service à l'humanité et s'intégrait fort bien à une philosophie humaniste et élitiste très répandue à l'époque. Propagée dans tous les établissements d'enseignement, cette philosophie contribuait à préparer le 
futur professionnel à un usage raisonnable de son autonomie et de son pouvoir.

En l'absence d'autres mécanismes pour contrôler les producteurs de services, les corporations professionnelles ont accompli leur tâche avec un certain succès mais non de façon totalement désintéressée : contribution à une standardisation des programmes de formation mais élévation constante et parfois superflue des exigences de scolarité et d'admission à la pratique; surveillance de la publicité mais élaboration de règles visant surtout l'élimination de la concurrence dite "déloyale " entre confrères; adoption de codes de déontologie afin de régir les relations des membres avec le public mais aussi les "bonnes relations » entre les membres.

\section{Le corporatisme professionnel dans une société en mutation}

L'industrialisation rapide de la société québécoise et la révolution technologique provoquée par la Seconde Guerre mondiale ont considérablement modifié les conditions de travail et les caractéristiques de la main-d'œuvre. Trois phénomènes, en particulier, retiennent l'attention. Ce sont : la salarisation de la main-d'œuvre, le morcellement des activités de travail dû à l'éclatement des champs de connaissances et l'intervention de l'État dans la production des services essentiels.

\section{a) La salarisation de la main-d'œuvre}

La prise en charge croissante des activités de travail par des organisations de grande dimension, entreprises privées ou publiques, a entraîné une salarisation graduelle de la main-d'œuvre. En effet, la concentration du capital ainsi que la complexité accrue des tâches et leur spécialisation ont profondément modifié le contexte économique qui permettait autrefois à un travailleur qualifié d'exercer individuellement son métier ou sa profession. La notion d'interdépendance s'est substituée à celle d'autonomie de sorte qu'un grand nombre de travailleurs qualifiés détenant des connaissances spécialisées sont devenus des salariés.

Ce processus de salarisation de la main-d'œuvre a considérablement réduit la contribution des corporations professionnelles au contrôle de la distribution des services professionnels. Car, devenus salariés, les travailleurs sont à la fois moins isolés et moins autonomes. Ils expriment habituellement leur solidarité à l'intérieur d'organisations syndicales expressément destinées à la défense de leurs intérêts propres et sont soumis à un contrôle hiérarchique formel, plus régulier et plus précis que le contrôle exercé par une corporation professionnelle auprès de ses membres de pratique privée. 
En fait, bien souvent, l'employeur, à ses propres fins, exécute de plus près, et de manière plus suivie que ne pourrait le faire une corporation professionnelle, des fonctions de discipline, d'évaluation de la compétence et de formation continue. Dans ce contexte, le mécanisme de la corporation professionnelle ne fait que s'ajouter à d'autres mécanismes visant la défense des intérêts des consommateurs et, à première vue, répond à des besoins de protection du public moins intenses et partiellement satisfaits.

Bref, si le développement des connaissances et l'industrialisation de la société ont multiplié et diversifié le nombre d'emplois exigeant des qualifications supérieures et le nombre de services offerts à la population, la salarisation de ces travailleurs a stabilisé la production et la distribution de ces services comme l'avait fait antérieurement le mécanisme de la corporation professionnelle pour les services offerts en pratique privée.

\section{b) Le morcellement des activités de travail et l'éclatement des champs de connaissances}

Le morcellement des tâches correspond à une division de plus en plus poussée du travail. Les professionnels peuvent de moins en moins prétendre offrir des services qui couvrent la totalité d'un champ de connaissances très étendu. Leur travail est souvent morcelé, spécialisé. Par ailleurs, le recrutement des travailleurs qualifiés repose autant sur le fait qu'ils ont acquis une compétence particulière dans une spécialité précise et qu'ils sont devenus experts dans tel secteur, telle méthode ou tel procédé, que sur le fait qu'ils détiennent un niveau de scolarité donné. Le champ d'activité du travailleur qualifié est de plus en plus spécifique.

II n'est donc plus nécessaire d'acquérir tout le savoir accumulé dans un champ de connaissances étendu pour offrir un service précis et bien défini. Une formation relativement peu étendue mais spécifique suffit pour poser des actes déterminés dans un champ d'activité restreint. Ainsi de nombreuses techniques d'intervention sont apparues dans des champs de connaissances auparavant occupés par les membres d'une seule corporation professionnelle, et il est courant de rencontrer, dans un même secteur d'activité, des équipes de travail constituées de personnes de formation diverses et de niveau de scolarité différent. Au morcellement des activités de travail correspond l'éclatement des champs de connaissances puisque l'activité de travail tend à être définie davantage en fonction de besoins spécifiques qu'en relation avec un champ de connaissances général.

Cette réduction des champs d'activité des travailleurs qualifiés facilite la surveillance de la main-d'œuvre dans les milieux salariés. Le 
contrôle hiérarchique s'exerce plus facilement auprès de travailleurs appelés à poser des actes précis, selon des méthodes éprouvées, dans des circonstances définies.

Toutes les activités de travail n'ont évidemment pas évolué au même rythme et certaines se prêtent beaucoup plus difficilement à un tel morcellement. II n'apparaît pas toujours possible, en effet, de fractionner un champ de connaissances, ou même, lorsque cela est possible, de cerner toutes les techniques naissantes avec suffisamment de précision pour les enfermer dans des définitions rigides. Ce ne serait d'ailleurs pas nécessairement souhaitable étant donné que ces techniques se chevauchent souvent les unes aux autres dans des secteurs d'activité connexes ou même se superposent à l'intérieur d'un même secteur. Néanmoins, les domaines sont de moins en moins nombreux où la corporation professionnelle autonome constitue le seul mécanisme de contrôle d'un producteur offrant des services complets à l'intérieur d'un champ de connaissances étendu et exerçant sa profession individuellement, en pratique privée.

\section{c) L'intervention de l'État dans la production des services essentiels}

Omniprésentes dans l'idéologie professionnelle, l'idée de « vocation " et celle $d^{\prime}$ " idéal de service " étaient en harmonie avec l'existence de besoins individuels auxquels on pouvait répondre par une action charitable. Les professionnels, médecins, avocats et autres, exerçaient une certaine forme de justice distributive en tenant compte, dans leurs relevés d'honoraires, de la capacité de payer du client et en acceptant généralement de rendre des services gratuitement à des personnes démunies. Cette attitude conforme aux idées courantes dans toutes les corporations professionnelles constituait une forme non négligeable d'autocontrôle des producteurs de services essentiels. Le corporatisme professionnel comblait ainsi un besoin social important.

Or, ce besoin est beaucoup moins pressant depuis la reconnaissance de certains droits sociaux fondamentaux - santé, logement, revenu minimum, etc. - et l'intervention de l'État dans la production des services essentiels. Cette intervention, en particulier, suit une tendance apparemment irréversible, vu la croissance des revenus publics et l'émergence de nouveaux besoins exigeant une action prioritaire de l'État.

Sous diverses formes - financement, réglementation, prise en charge ou production directe des services - l'intervention de l'État dans le domaine des services professionnels est devenue considérable : plus ou moins directement, l'État est le principal employeur 
des professionnels ou le principal client des services professionnels offerts en pratique privée. Ce contexte qui modifie sensiblement les rapports entre professionnels et clients rend moins essentielle la constitution d'une nouvelle corporation professionnelle dans un secteur donné, d'autant plus que d'autres mécanismes peuvent lui être avantageusement substitués. L'État peut en effet adopter des lois lui permettant de réglementer directement une activité de travail et de délivrer lui-même des permis de pratique à ceux qui l'exercent. II peut aussi adopter diverses lois de caractère plus général qui viennent régir plus indirectement la distribution des services professionnels : Loi sur les services de santé et les services sociaux ${ }^{5}$, Loi sur la protection du consommateur ${ }^{6}$, Loi (fédérale) relative aux enquêtes sur les coalitions ${ }^{7}$, etc.

Considéré séparément, chacun de ces phénomènes - salarisation de la main-d'œuvre, morcellement des activités de travail et intervention de l'État - réduit sensiblement l'apport prévisible du corporatisme professionnel à de nouveaux secteurs d'activité. À plus forte raison, leur développement simultané dans les dernières décennies incite à une grande prudence dans l'appréciation des besoins de constitution de nouvelles corporations professionnelles. $C^{\prime}$ est ce qui explique pourquoi, depuis la dernière guerre, le législateur s'est montré réticent à constituer de trop nombreuses corporations professionnelles et pourquoi, lorsqu'il l'a fait, il a préféré octroyer des titres réservés plutôt que des exercices exclusifs.

Le degré d'insertion des corporations professionnelles dans les milieux salariés paraît en particulier soulever plusieurs interrogations. Certes, ni la salarisation des professionnels, ni l'intervention de l'État dans la distribution des services au public n'empêchent, en soi, une corporation professionnelle de veiller à la qualité des services offerts par ses membres et de protéger ce même public. Cependant, I'analyse effectuée par I'Office révèle que, sur ce plan, la plupart des corporations dont la majorité des membres sont des employés d'entreprises, d'établissements ou d'institutions ne se sont pas acquittées de leur tâche de façon convaincante.

En résumé, l'analyse permet de constater que :

1. Le corporatisme professionnel s'insère dans un contexte historique particulier de la réglementation de certaines activités de travail et n'est pas inhérent à la nature de ces activités.

2. Ce mécanisme de réglementation n'est pas indéfiniment extensible. Créé dans un contexte où il n'existait pas d'autre formule de contrôle de la distribution de certains services essentiels, il ne peut plus, désormais, se justifier de la même façon, dans la mesure où l'évolution des activités de travail et l'intervention de l'État dans la 
production des services contribuent à stabiliser le marché et à encadrer efficacement les producteurs.

3. Dans ce nouveau contexte, il devient plus difficile de fonder sur la seule autogestion des producteurs une politique générale de protection du public ou de protection des consommateurs des services concernés.

\section{Méthode utilisée}

\section{Les entrevues}

Le programme d'entrevues que nous avons entrepris avait pour but d'évaluer la perception qu'ont les praticiens sociaux de la question de la morale professionnelle, de jauger leur familiarité avec leur code de déontologie et de vérifier dans quelle mesure il est perçu comme moyen de contrôle sur la pratique professionnelle. Notre intention était, en fait, de jeter un coup de sonde du côté des praticiens sociaux, coup de sonde que nous avons voulu suffisamment rigoureux pour servir utilement nos fins de recherche.

\section{La population interrogée}

Au total nous avons interrogé 142 praticiens sociaux. Trois variables ont guidé le choix des sujets devant constituer notre population interrogée.

1. Variable géographique : la province de Québec;

2. Variable linguistique : la langue française;

3. Le type de pratique.

Nous avons distingué essentiellement la pratique privée de la pratique dans une organisation et à cette fin nous avons retenu les catégories suivantes :

a) pratique en bureau privé;

b) pratique au sein d'une organisation dans le secteur public ou parapublic.

Sans être scientifiques, ces critères nous permettaient de varier notre population tout en restant à l'intérieur d'une aire géographique facilement accessible. La distribution de nos sujets effectivement interviewés s'est donc faite comme l'indique le tableau 1. 


\section{Tableau 1 : Répartition des praticiens sociaux interrogés selon} les régions administratives du Québec

\begin{tabular}{|llr|}
\hline \multicolumn{2}{|l|}{ Régions administratives } \\
01 & Bas-Saint-Laurent et Îles-de-la-Madeleine & 6 \\
02 & Saguenay-Lac-Saint-Jean & 1 \\
03 & Québec & 128 \\
04 & Centre du Québec & 1 \\
05 & Estrie & 1 \\
$06 \mathrm{~A}$ & Montréal métropolitain & 1 \\
$06 \mathrm{C}$ & Richelieu & 1 \\
07 & Outaouais & 1 \\
08 & Nord-Ouest & 1 \\
09 & Côte-Nord & 1 \\
& & 142 \\
\hline
\end{tabular}

\section{Le schéma d'entrevue}

Les interviewers ont utilisé pour leurs entrevues un schéma simple pour encadrer leurs discussions avec les sujets interrogés. Ce schéma (voir annexe 1), qui n'était pas un questionnaire comme tel, comportait quatre sections :

1. une section sur les rapports praticien social - code de déontologie, où l'on cherchait à évaluer la connaissance que le praticien social avait de son code et l'utilisation qu'il en faisait;

2. une section sur les rapports profession - code de déontologie où I'on demandait au praticien social de nous informer sur ce que faisait sa profession en matière d'éthique;

3. une section sur les rapports client - code de déontologie où l'on demandait à l'interrogé d'aborder la question éthique en fonction de ses rapports avec ses clients et, enfin;

4. une section "Divers" où l'on cherchait à obtenir des renseignements sur quelques points précis non couverts par les sections précédentes, tels que " inventaire des actes dérogatoires à la personne professionnelle, évaluation de la pertinence de certains articles du code ", etc.

Le schéma n'avait pas pour but de vérifier des hypothèses ou de mesurer avec précision des variables, mais tout simplement de recueillir ce que les praticiens sociaux pensent de la question déontologique, tout en essayant d'évaluer leur connaissance du code de déontologie. 


\section{Le déroulement des entrevues}

La liste des sujets à rencontrer fut constituée au hasard à partir des listes des superviseurs de stages de l'École de service social de I'Université Laval.

Les entrevues, effectuées par des interviewers, ont eu lieu entre le 15 janvier et le 30 avril 1990. Les interviewers ont fait une entrevue par jour et ont rédigé leur compte rendu la journée même à l'aide de l'enregistrement de la conversation. Ils ont ajouté à leurs notes des commentaires sur le climat de l'entrevue et toute information permettant d'évaluer la crédibilité des répondants.

\section{L'analyse du matériel}

Pour simplifier l'analyse du matériel volumineux ainsi accumulé, nous avons, après avoir éliminé les éléments non significatifs des entrevues, construit une grille de codification des réponses obtenues pour permettre un traitement mécanique des entrevues.

Il a ainsi été possible de procéder à une analyse quantitative des résultats d'entrevue en ayant recours aux ordinateurs du Centre de traitement de l'information de l'Université Laval. À cette analyse quantitative est venue s'ajouter une analyse qualitative.

\section{Analyse des résultats}

II est évident qu'on ne peut préjuger des comportements des praticiens sociaux en se contentant d'examiner le contenu de leur code de déontologie. Rien ne démontre, à première vue, que ce code suscite des comportements conformes aux règles qu'il énonce. Le passage du principe à la réalité n'est pas automatique, cela va de soi.

II n'est cependant pas facile de mesurer avec précision la conformité des comportements des praticiens sociaux avec les règles de conduite édictées par le code qu'ils prétendent respecter. II faudrait, pour cela, entreprendre des analyses en profondeur, ce qui, pour nous, ne s'avérait pas possible. Nous avons plutôt opté pour une démarche simple dont le but n'était que de fournir une première évaluation de la perception qu'ont les praticiens sociaux, dans leur pratique quotidienne, de la question de l'éthique professionnelle.

Nos objectifs étaient essentiellement de sonder le milieu des praticiens sociaux pour recueillir d'une part des informations sur les comportements des praticiens sociaux (connaissance, usage et diffusion des règles de conduite) et, d'autre part, des opinions sur le 
contenu, l'efficacité, la nécessité de cet instrument d'autocontrôle qu'est le code de déontologie.

Nous avons donc procédé à une série d'entrevues auprès de 142 praticiens sociaux. La population interrogée a été décrite plus haut. Cet échantillon aléatoire n'est pas représentatif, au sens statistique du terme, du groupe étudié, mais il nous apparaît suffisant pour donner des indices intéressants sur les comportements et attitudes des praticiens sociaux. II est sûr qu'il faut dès maintenant émettre des réserves sur les interprétations à donner aux réponses qui nous ont été communiquées. D'abord, le fait que notre population soit aussi petite limite les possibilités d'établir des corrélations entre les variables disponibles sur les caractéristiques personnelles et professionnelles des sujets interrogés (âge, sexe, type et lieu de pratique, années d'expérience) membres ou non de la Corporation et les réponses fournies à nos questions. C'est pourquoi nous n'avons retenu, pour étude, que les données les plus globales pour l'ensemble des praticiens sociaux représentés dans notre échantillon.

Deuxièmement, les entrevues que nous avons réalisées ont été faites à partir d'un canevas ou schéma d'entrevue composé en bonne partie de questions ouvertes. Ce n'est qu'a posteriori que nous avons codifié l'ensemble des réponses fournies et créé des catégories pour les classer. En d'autres termes, lorsqu'on demandait, par exemple, à un praticien social d'évaluer l'efficacité de son code de déontologie, il n'avait pas à choisir entre deux ou plusieurs réponses prédéfinies; plutôt, il répondait spontanément et ce n'est qu'en recensant les différentes réponses faites à cette question que nous avons, après coup, créé des catégories de classement. Cette façon de procéder ne permet pas, bien sûr, d'atteindre la précision que permet un questionnaire; cependant, elle offre l'avantage de fournir des réactions spontanées qui souvent peuvent être particulièrement révélatrices.

Ces réserves étant faites, nous allons passer en revue les réponses obtenues aux cours des entrevues. Nous avons regroupé autour de sept thèmes les questions et réponses. Ce sont respectivement :

- Connaissance du code.

- Formation déontologique.

- Usage du code.

- Diffusion du code.

- Niveau d'information du client.

- Contenu du code.

- Efficacité et nécessité du code. 


\section{La connaissance du code}

Quand on leur demande d'évaluer leur connaissance du contenu de leur code de déontologie, la majorité des praticiens sociaux répondent qu'ils en ont une connaissance globale, c'est-à-dire qu'ils en connaissent le sens général, qu'ils en ont intégré les principes, en somme qu'ils l'ont intériorisé. Ainsi un praticien social de Québec répond:

Je n'ai jamais appris mon code par cœur, mais je le connais assez bien.

Près du quart des répondants signalent ne connaître le contenu de leur code que par un article ou une section en particulier. Parmi ceux qui disent le connaître " très bien » $(11,4 \%)$, on retrouve habituellement des individus qui ont été mêlés de près aux affaires de leur corporation professionnelle et qui à ce titre ont dû $s^{\prime} y$ intéresser.

Enfin, 10,7 \% des répondants affirment carrément ne pas connaître du tout le contenu de leur code de déontologie.

Sauf exception, les praticiens sociaux ne semblent pas connaître leur code de façon précise. Pour la plupart, le code ne fait qu'énoncer des principes généraux qui relèvent, de toute façon, du bon sens et de la logique. Sans connaître le contenu exact de ces énoncés, les praticiens sociaux disent cependant bien connaître ce qui leur est permis et ce qui leur est défendu :

Je connais assez mal les articles spécifiques de notre code. Je sais toutefois ce qu'il nous est permis de faire, de même que ce qui nous est défendu (praticien social de Beauce).

Ce genre de remarque nous a été fait à de nombreuses reprises.

\section{La formation déontologique}

Nous avons demandé aux répondants dans quel contexte ils avaient entendu parler d'éthique professionnelle dans le cours de leur formation. Parmi eux, $22 \%$ disent avoir suivi des cours de déontologie, $45 \%$ ont vaguement entendu parler d'éthique durant leur formation et $43 \%$ n'en ont même jamais entendu parler.

II n'est donc pas étonnant que la connaissance du code de déontologie chez les praticiens sociaux soit généralement globale et vague, puisqu'une bonne proportion d'entre eux n'ont pratiquement pas entendu parler de déontologie pendant leur formation professionnelle. Une enquête sommaire que nous avons faite auprès de la Corporation, de même que des départements et des écoles professionnelles, nous a confirmé que la formation déontologique était à peu près inexistante dans la plupart des $\operatorname{cas}^{8}$. 


\section{Usage du code de déontologie}

La référence formelle au code de déontologie est chose exceptionnelle; la grande majorité des répondants ne se réfèrent pas directement au code. Plusieurs répondent qu'ils n'ont pas besoin de consulter formellement leur code parce qu'ils l'ont intériorisé :

(je ne m'y réfère) pas dans le sens que ça m'oblige à sortir le code de ma bibliothèque pour le consulter. Je me réfère à ce que j'ai en tête (praticien social, Montréal). C'est plutôt une référence à des principes qu'à des règles explicites, mises en forme dans un texte quelconque (praticien social, Québec).

Parmi les répondants, $63 \%$ se réfèrent peu souvent, sinon jamais, à leur code de déontologie. Certains spécifient que le problème ne se pose pas, qu'on n'a pas besoin de recourir à un tel instrument ou encore qu'il suffit d'un peu de bon sens pour savoir ce que l'on doit faire ou pas.

Bien peu, en fait, voient dans le code un outil de référence qui puisse permettre de résoudre des problèmes dans le cadre de la pratique quotidienne. On préfère plutôt recourir à l'avis des collègues ou demander des informations à un organisme professionnel, la corporation, le syndicat, etc.

\section{La diffusion du code}

Le praticien social informe-t-il ses clients de la protection que leur accorde son code de déontologie ? On note que $41 \%$ des personnes interrogées disent I'avoir déjà fait à I'occasion, 51 \% répondent ne l'avoir jamais fait et, enfin, $8 \%$ ne savent pas ou ne répondent pas.

Parmi ceux qui ont répondu oui à cette question, un bon nombre se réfèrent à des points précis de leur code de déontologie; le point le plus souvent évoqué est celui de la confidentialité. Quand on informe son client, en général, c'est pour l'assurer que ce qui est porté à la connaissance du professionnel au cours de sa relation avec lui constitue l'objet du " secret professionnel ». Les praticiens sociaux interrogés disent, dans une proportion de $55 \%$, avoir déjà informé leurs clients du contenu de leur code et tous ont spécifié qu'il s'agissait d'information à propos de la confidentialité. Un praticien social, quant à lui, nous décrit comment certains de ses clients envisagent cette question de la confidentialité :

... ce qui les inquiète le plus c'est le Régime d'aide sociale. Mon expérience, moi, c'est quand les gens ont manifesté une inquiétude envers ça. Ils ont peur qu'à l'aide sociale, il y ait des gens qui soient au courant qu'ils sont venus consulter un praticien social (Québec). 
Devant des craintes de cette nature il convient de se poser la question de l'efficacité des règles ayant trait au secret professionnel; c'est le problème de l'extension des règles de confidentialité à tous ceux qui peuvent prendre connaissance d'informations livrées au praticien social sous le sceau du secret.

Nous avons aussi retrouvé, parmi ceux qui ont répondu oui à notre question, des praticiens sociaux qui disent avoir mis leurs clients au courant du contenu de leur code pour se protéger euxmêmes contre des demandes abusives d'un client. C'est un praticien social en protection de la jeunesse qui, par sa réponse, illustre le mieux ce point de vue :

On fait connaître aux clients certains articles qui les protègent (secret professionnel) mais, vu notre situation, on explique plus souvent le contenu du code pour se protéger contre les représailles des clients. Le code sert beaucoup dans ces moments-là. On aimerait bien qu'il soit encore plus précis (praticiens sociaux, Québec).

II reste cependant que moins de la moitié des praticiens sociaux interrogés font connaître leur code à leur client et pas un ne nous a dit le faire régulièrement. La très grande majorité des réponses ont consisté en des "je l'ai déjà fait ", " ça m'est arrivé à l'occasion ", etc. Cela indique, pour le moins, que ce n'est pas une pratique établie que d'informer le client des règles que la profession impose à ses membres.

\section{Niveau d'information du client}

À la question "Vos clients connaissent-ils l'existence de votre code de déontologie ? ", $90 \%$ des personnes interrogées n'hésitent pas à répondre que, en règle générale, leurs clients ne connaissent même pas l'existence de leur code. Les autres $10 \%$ nous ont dit que le client soupçonne qu'être professionnel cela suppose qu'on est soumis à certaines règles.

Cela n'empêche pas $82,1 \%$ des répondants de croire que, en dépit d'une ignorance généralisée, le client est bien protégé par un code de déontologie, alors que $8,8 \%$ pensent le contraire et que $9,1 \%$ des personnes interrogées n'ont pas répondu. Notons que $50 \%$ estiment que leurs clients ne sont pas suffisamment informés du contenu du code, $30 \%$ croient que oui et $20 \%$ n'ont pas répondu.

Ainsi, même dans le cas où le client ignore la protection que lui offre un code de déontologie professionnelle, cette protection reste quand même valable aux yeux des praticiens sociaux. 


\section{Le contenu du code}

Ici nous avons voulu savoir des praticiens sociaux ce qu'ils considéraient comme "actes dérogatoires à l'honneur professionnel " méritant d'être réprouvés par un code de déontologie. La question était ouverte et, en réponse, les actes désignés comme dérogatoires chez les praticiens sociaux sont principalement "le non-respect du secret professionnel » et " la mauvaise qualité du service rendu ».

Parmi les autres actes dont il fut fait mention, citons "le fait de dénigrer un collègue " et " le fait de fermer un dossier sans raison ".

À l'examen des préoccupations des praticiens sociaux, on constate qu'à première vue ces derniers semblent plus portés à valoriser la qualité du service à rendre.

Outre la question ayant trait aux actes considérés comme dérogatoires à l'honneur professionnel, nous avons demandé aux interviewés s'ils croyaient leur code adapté à la réalité actuelle : $49 \%$ ne savent pas ou ne répondent pas, $34 \%$ croient que oui, $15 \%$ répondent plus ou moins et seulement $2 \%$ croient que non. Cette réponse est difficile à interpréter : en fait, ce qui est intéressant, c'est que $49 \%$ ne sont pas en mesure de répondre à une telle question, probablement à cause d'une méconnaissance du code.

\section{Efficacité et nécessité du code}

Globalement, quand on demande aux praticiens sociaux d'évaluer l'efficacité de leur code à résoudre les problèmes d'éthique qui se posent dans le cours de leurs activités, $33 \%$ croient qu'il est efficace, $17 \%$ ne savent pas ou ne répondent pas et $50 \%$ croient, à divers degrés, qu'il n'est pas efficace.

Parmi les réponses négatives à cette question, un bon nombre d'entre elles faisaient état de problèmes précis que le code parvenait mal à régler; on nous signalait des problèmes particuliers aux praticiens sociaux, comme les questions de la comparution en cour à titre d'expert et de propriété des dossiers.

On constate cependant que même si les praticiens sociaux ne croient pas dans une forte proportion à l'efficacité de leur code, une très forte majorité d'entre eux croient toujours en sa nécessité. À la question "Croyez-vous qu'un code de déontologie soit nécessaire à votre profession ? ", $81 \%$ des interviewés répondent oui; parmi les $13,5 \%$ qui ne croient pas le code nécessaire, la plupart assortissent leur réponse d'un commentaire du genre "On peut s'en passer, le gros bon sens suffit».

II ne faut pas, par ailleurs, croire que tous les praticiens sociaux qui estiment le code de déontologie nécessaire le font pour les mê- 
mes raisons : les quelques exemples de réponses qui suivent illustrent ce que nous venons de dire :

Le fait d'avoir un code de déontologie et un contrôle rassure le public du côté de la qualité du service qu'on leur rend.

Malheureusement, c'est nécessaire un code; il faut indiquer aux membres les limites qui ne doivent pas être dépassées et surtout, il faut indiquer aux membres les sanctions qui leur seront imposées.

Un code, c'est nécessaire. On a besoin de règles pour dicter des conduites entre collègues. II faut protéger la profession et se protéger soi-même.

Un code c'est nécessaire, parce qu'il faut protéger les membres par une réglementation qui nous donne des limites claires dans l'exercice de notre pratique.

Oui, je crois à la nécessité du code à $100 \%$, pas tellement pour les membres, mais pour le public.

Le code apparaît donc nécessaire, pour plusieurs, pour protéger à la fois le praticien social et le client.

On peut donc retenir que malgré le fait qu'il leur apparaisse peu efficace, les praticiens sociaux estiment leur code de déontologie nécessaire. Il y aurait peut-être lieu de miser sur ce sentiment pour lancer, dans la profession, le débat autour des questions déontologiques.

\section{Conclusion}

Nous retiendrons de ces entrevues qu'en général les praticiens sociaux connaissent leur code de déontologie de façon superficielle, et que cela tiendrait au fait que leur formation éthique formelle est limitée. Ils font plutôt rarement usage de leur code, ne le font pas connaître de façon spécifique à leurs clients. Enfin, même s'ils doutent de son efficacité, ils continuent de croire à la nécessité du code de déontologie.

Malgré les réserves que nous avons faites à propos de notre méthode de travail, nous croyons que ce coup de sonde dans le milieu des praticiens sociaux nous permet $d^{\prime}$ avoir en main un portrait du rapport praticien social - éthique qui ne nous semble pas s'éloigner de façon importante de la réalité.

En terminant, nous pouvons affirmer, à partir des résultats obtenus, qu'il est nécessaire d'améliorer la formation sur les principes éthiques et leur application dans la pratique. II est du devoir de la Corporation, intervenant conjointement avec les universités et les écoles professionnelles, de veiller non seulement à la formation déontologique de base, mais aussi au recyclage déontologique des praticiens sociaux en exercice. 


\section{Notes et références}

${ }^{1}$ Voir à l'annexe 2 la liste des 155 étudiantes et étudiants participants.

${ }^{2}$ Par praticiens sociaux, nous entendons tous les diplômés universitaires en service social membres ou non de la Corporation professionnelle des travailleurs sociaux du Québec.

${ }^{3}$ Jean-Paul Rouleau, Gilles Dussault, Michel Duchesneau, Alain Gagnon, Danièle Massé et Louis O'Neil (1977). La déontologie professionnelle au Québec, Institut supérieur des sciences humaines, Québec, 291 p.

${ }^{4}$ Office des professions du Québec (1976). " L'activité des corporations professionnelles vue dans une perspective historique ", L'évaluation du professionnalisme au Québec, p. 50-55.

${ }^{5}$ L.Q. 1971, chap. 48.

${ }^{6}$ L.Q. 1971, chap. 74.

7 L.Q. 1970, chap. C-23, modifié par S.C. 1974-1975, chap. 76. Cette modification a étendu l'application de cette loi au secteur des services.

${ }^{8}$ Seule l'École de service social de l'Université Laval offre un cours de déontologie professionnelle dans les départements et écoles de service social francophones du Québec.

\section{Annexe 1 Schéma d'entrevue}

A. Le professionnel et le code de déontologie

1. Votre profession vous propose-t-elle un code de déontologie ?

2. En connaissez-vous le contenu ?

- très bien

- les principales règles

— un peu

- pas du tout

3. Au cours de votre formation, avez-vous entendu parler de déontologie professionnelle?

- suivi des cours

- lu des ouvrages

- conférences

- etc.

4. Dans l'exercice quotidien de votre profession, rencontrezvous des situations qui nécessitent que vous vous référiez à votre code de déontologie?

- régulièrement, toutes les semaines

- 1 ou 2 fois par mois

- jamais

- ne sait pas 
5. En général, vos collègues connaissent-ils l'existence d'un code de déontologie professionnelle dans leur profession?

En connaissent-ils le contenu ?

6. Les praticiens de votre profession recourent-ils à leur code de déontologie?

À quelle fréquence?

À quelles règles surtout?

- secret professionnel

- relations avec les collègues

- publicité

- honoraires

- etc.

7. Vous est-il déjà arrivé de faire connaître à vos clients le contenu de votre code de déontologie pour les rassurer sur la protection qu'il prévoit pour eux ?

8. Croyez-vous que vos collègues le font?

B. Le client et le code de déontologie

1. En général, vos clients connaissent-ils l'existence de votre code de déontologie professionnelle?

2. Avez-vous eu connaissance de cas où des clients auraient invoqué des règles de ce code pour se plaindre, pour obtenir un meilleur service, etc. ?

3. Selon vous, le client est-il bien protégé par un code de déontologie?

Comment?

4. Vos clients sont-ils suffisamment informés du contenu de votre code?

C. La profession et le code de déontologie

1. Dans ses activités courantes, votre corporation professionnelle fait-elle place aux questions d'éthique professionnelle?

- Colloques, comités, congrès, articles de revue...

2. Votre corporation professionnelle a-t-elle souvent recours au code de déontologie?

- ... à des fins disciplinaires auprès de ses membres?

- ... auprès de l'État ou de toute autre organisation, en vue de protéger ses membres?

- ... pour protéger des clients (ex. : secret professionnel) ?

- À quelle fréquence?

— Quelles sont les règles le plus souvent utilisées à ces fins? 
3. L'application du code de déontologie est-elle surveillée ?

- Par qui ? - comité de discipline ?

- Comment ? - plaintes, enquêtes?

4. Le contenu du code fait-il l'objet de révisions périodiques ?

$\mathrm{Y}$ a-t-il eu des changements dans son contenu depuis sa première rédaction?

Si oui, lesquels?

Selon vous, votre code de déontologie est-il adapté à la réalité actuelle?

5. Quelle place votre corporation professionnelle fait-elle à la "protection du public " dans l'ensemble de ses objectifs?

6. Que fait la profession pour diffuser les règles déontologiques qu'elle se donne?

- auprès de ses membres ?

- auprès de ses clients?

— auprès du public, en général ?

D. Autres questions

1. Quels sont, selon vous, les actes qui devraient être considérés comme dérogatoires à l'honneur professionnel et être énumérés dans un code de déontologie ?

2. Le code actuel permet-il de faire face à tous les problèmes d'éthique?

3. Y aurait-il lieu d'y ajouter ou d'en retrancher des articles ?

4. Croyez-vous à la nécessité d'un tel code ?

Etc.

\section{Annexe 2}

Liste des 155 étudiantes et étudiants participants

Allaire, Richard

Ambroise, Valérie

Audet, Susan

Baker, André

Barry, Bruno

Beaudoin, Manon

Beaulieu, Hélène

Bédard, Marie-Claude

Bédard, Yves
Bégin, Kathleen

Bernard, Nicole

Bernier, Sylvie

Bérubé, Nancy

Bilodeau, Gina

Binette, Geneviève

Blais, Chantal

Boivin, Kathy

Boivin, Manon
Bolduc, Johanne Bouchard, Martine Boucher, Linda Boulianne, Diane Bourbonnais, Sylvie Brousseau, Michel Brousseau, Sophie Burns, Michael Caron, Julie 


\begin{tabular}{|c|c|c|}
\hline Castonguay, Anne & Gendron, Claire & Nadeau, Gaétan \\
\hline Castonguay, Josée & Gendron, Denis & Normand, Hélène \\
\hline Chabot, Céline & Gingras, Sylvie & Ntoutoume Emane, \\
\hline Charest, Marthe & Girard, Sylvie & André \\
\hline Chevalier, Annie & Gobeil, Marie-Josée & Ouellet, Sylvie \\
\hline Clermont, Julie & Guay, Daniel & Paré, Chantale \\
\hline Côté, Caroline & Guimond, Martine & Patry, Marlène \\
\hline Côté, Caroline & Jutras, Anne & Payeur, Angèle \\
\hline Côté, Chantal & Kelly, Jane & Pelletier, Annie \\
\hline Couture, Brigitte & Labbé, Nancy & Pelletier, Marc \\
\hline Dallaire, Manon & Labelle, Manon & Perron, Claudie \\
\hline Danis, Jocelyne & Laberge, Andrée & Pleau, Maryse \\
\hline Day, Sandra & nbe, Raynald & Poirier, Lynda \\
\hline Deschênes, François & Lafrance, Louise & Poliquin, Ginette \\
\hline ochers, Bernard & lesse, & Pomerleau, Hélène \\
\hline Dion, $\mathrm{Ar}$ & Marjolaine-Joan & in, Patricia \\
\hline Dion, Manon & the, Sylvie & Pouliot, Nancy \\
\hline Doré, Nathalie & Landry, François & ne, Nathalie \\
\hline Drouin, Nicole & nte, Louis & Richer, Johanne \\
\hline in-St-Gelais, F. & Lapointe, Marie & Roberge, Marie-Fran \\
\hline Denise & ite, Maryse & Rochette, Doris \\
\hline ur, Carole & Laroche, Nathalie & Romero, Maria-Éléna \\
\hline Dufour, Renée & che, Bruno & Roy, André \\
\hline Dufresne, Nathalie & Lemay, Marie-Claude & Jean-Louis \\
\hline ay, Sylvie & Suzie & Savard, Lynda \\
\hline Dumas, Marie-Claude & Leblanc, Pierrette & ie, Louise \\
\hline ult, Sonia & rc, Julie & Simard, Hélène \\
\hline Fallu, Caroline & Leduc, Louis & d, Robert \\
\hline n, Sylvie & , Nathalie & St-Amand, Lynda \\
\hline Flaschner, Nathalie & e, Martine & laire, Dominique \\
\hline , Judith & Nancy & Sullivan, Linda Ann \\
\hline Fortin, Lisa & Malo, Luc-Antoine & , Mireille \\
\hline , Marie & tte, Diane & Tardif, Suzanne \\
\hline Fréchette, Linda & een, Anne & Thivierge, Natalie \\
\hline tte, Kathy & is, Yvan & Tousignant, Anne \\
\hline Gaboury, Marie- & Martel, Lyne & Tremblay, Diane \\
\hline Claude & eau, Donald & Tremblay, Dom \\
\hline Gagné, Maryse & Mathieu, Anie & Tremblay, Mathilde \\
\hline on, Nathalie & els, Susy & Trudel, Catherine \\
\hline Gagnon, Sylvie & Michaud, Éric & Turbide, Linda \\
\hline Gaudreau, Johanne & Moore-Turgeon, & Turgeon, Liliane \\
\hline hond, Line & Marcelle & Turgeon, Martine \\
\hline ier, Danielle & au, Nicol & Vachon, Denise \\
\hline Gauthier, Sophie & Morin, Sylvie & Vaillancourt, Sonya \\
\hline
\end{tabular}

